\title{
Are plasma concentrations of tamoxifen active metabolites sufficient to ensure therapeutic efficacy for tamoxifen treated women with breast cancer in Poland?
}

\author{
Ewa E. Hennig
}

\begin{abstract}
Tamoxifen is the most commonly used drug for treating those patients with breast cancer who are oestrogen receptor positive. The main active metabolite of tamoxifen is (Z)-endoxifen whose therapeutic efficacy depends on its plasma concentration. A therapeutically effective threshold level has indeed been defined for (Z)-endoxifen above which the breast cancer relapse rate is significantly reduced. Such steady-state concentrations are conditional on gene polymorphism, principally cytochrome P450 2D6 (CYP2D6), that modulates the activity of the encoded enzymes that convert tamoxifen to its active metabolites. This drug's metabolism however may become significantly altered when other medication is concomitantly taken, such as selective serotonin reuptake inhibitors, which inhibit CYP2D6. A recent study have demonstrated that the majority of tamoxifen treated women with breast cancer in Poland, may not in fact attain the therapeutic threshold levels of (Z)-endoxifen. In such cases, personalising optimal treatment should be based on direct monitoring of steady-state plasma concentrations of tamoxifen and its metabolites, which can thereby significantly improve therapeutic efficacy.
\end{abstract}

NOWOTWORY J Oncol 2016; 66, 4: 307-311

Key words: tamoxifen, CYP2D6 genotype, endoxifen concentration, breast cancer

\section{Introduction}

Tamoxifen is one of the most widely used drugs in those patients with breast cancer expressing oestrogen receptors, and is proscribed both in primary and adjuvant therapy. It is also recommended for breast cancer prevention in high-risk carriers bearing mutations in the BRCA1 and BRCA2 genes [1]. This drug belongs to the group of selective oestrogen receptor modulators which inhibit tumour progression by blocking oestrogen binding to its receptor. When used in adjuvant therapy over 5 years, tamoxifen reduces the annual recurrence rate of the disease and mortality rate within the first 15 years, by respectively nearly $40 \%$ and $30 \%$ [2]. Prolonging the therapy to 10 years appears to be even more beneficial by further reducing the risks of recurrence and mortality by respectively another $29 \%$ and $25 \%$ [3]. However, the effectiveness of treatment greatly varies and disease recurs in $30-50 \%$ of patients that receive tamoxifen as adjuvant therapy [2]. Pure tamoxifen, as the pro-drug form, has little binding affinity towards the oestrogen receptor and hence its therapeutic efficacy is largely dictated by the action of enzymes that convert the pro-drug into derivatives that demonstrate up to a 100-fold greater anti-oestrogenic activity $[4,5]$. It becomes metabolised in the liver, primarily by the large family of cytochrome $\mathrm{P}_{450}$ (CYP) enzymes; with CYP2D6, CYP3A4, CYP3A5 and CYP2C19 being the most prominent. Phase II drug metabolising enzymes such as sulfotransferases (SULTs) and UDP-glucuronosyltransferases (UGTs), continue on the metabolic conversions to more hydrophilic forms thereby facilitating excretion from the body. The individual enzymes metabolising tamoxifen however, exhibit variable activities which are related to the polymorphic sequence variants of the genes that encode them.

Department of Gastroenterology, Hepatology and Clinical Oncology, Medical Center for Postgraduate Education; Department of Genetics, Maria Sklodowska-Curie Memorial Cancer Center and Institute of Oncology, Warsaw, Poland 


\section{Active metabolites of tamoxifen}

Hitherto, there have been at least thirty six phase I metabolites of tamoxifen described [6, 7]. Two main metabolic pathways lead to the formation of the $\mathrm{N}$-desmethyl-tamoxifen metabolite, which is found at the largest concentrations in plasma from treated patients, as well as 4-hydroxy-tamoxifen. These are then converted to 4-hydroxy- $N$-desmethyl-tamoxifen; termed as endoxifen [8]. Both 4-hydroxy-tamoxifen and endoxifen exhibit a 30 to 100 times higher affinity to the oestrogen receptor, and suppression of oestrogen-dependent proliferation of the human breast cancer MCF7 cells, when compared to $\mathrm{N}$-desmethyl-tamoxifen or the parent tamoxifen $[4,5]$. In chemical terms, tamoxifen occurs in its pure form as the (Z)-isomer, thus its metabolites are mainly of this form [9]. Those derivatives of tamoxifen which most highly inhibit the oestrogen receptor are the (Z)-isomers of endoxifen and 4-hydroxy-tamoxifen with IC50 values (half maximal inhibitory concentration) of respectively $3 \mathrm{nmol} / \mathrm{L}$ and $7 \mathrm{nmol} / \mathrm{L}[6]$.

\section{CYP2D6 polymorphism and tamoxifen metabolism}

The key enzyme responsible for converting tamoxifen into 4-hydroxy-tamoxifen and endoxifen is CYP2D6 [8]. It also exhibits the greatest polymorphic variability amongst the enzymes metabolising tamoxifen. Currently over 140 genetic variants (alleles) of CYP2D6, have been so far described, many of which are linked to a lack of, or decreased activity of the enzyme that it encodes for (based on http://www.cypalleles.ki.se/cyp2d6.htm). CYP2D6 function (CYP2D6 phenotype) results from the specific combination of the two alleles of the encoding gene (CYP2D6 genotype). Depending on their CYP2D6 genotype, every population member is classified according to four phenotype groups; poor metabolisers (PM), intermediate metabolisers (IM), normal - extensive metabolisers (EM) or ultra-rapid metabolisers (UM) [10]. The global occurrence of particular CYP2D6 alleles shows considerable ethnic variability, depending on the geographic region and population [11].

Tamoxifen treated women of the IM or PM phenotype are shown to have plasma concentrations of (Z)-endoxifen up to $75 \%$ lower than the normal EM phenotype [12-15]. Furthermore, the concentration of this metabolite increases with increasing numbers of CYP2D6 alleles encoding the functioning enzyme $[6,15]$. Likewise the drug's therapeutic efficacy, as measured by the disease-free survival period, demonstrates that those patients with impaired tamoxifen metabolism may not receive the full benefits of such treatment as compared to patients with the fully functional enzyme [16]. Ensuing attempts for CYP2D6 genotyping to predict the effectiveness of treatment has however not yet provided full and unequivocal outcomes and is still not recommended for routine testing [17-19].

\section{Therapeutic concentrations of endoxifen}

It is widely recognised that (Z)-endoxifen is the primary active metabolite of tamoxifen which is ultimately responsible for the drug's therapeutic efficacy. Its anti-oestrogenic properties are similar to (Z)-4-hydroxy-tamoxifen, nonetheless its plasma concentration in tamoxifen treated women is several times higher $[5,12,20]$. An endoxifen dose-dependent degradation of the oestrogen receptor has been observed [21], together with global gene expression induced by oestrogen in MCF7 breast cancer cells [22], and tumour growth inhibition in a MCF7 xenograft bearing mouse model [23]. Importantly, such effects were either absent or only slightly noticeable at those levels of endoxifen concentrations found in women with the CYP2D6 PM phenotype.

Amongst all the tamoxifen metabolites studied only endoxifen, up till now, has demonstrated a relationship between plasma concentrations in treated women with a corresponding therapeutic response [13,24, 25]. Recurrence rates were $26 \%$ lower in patients with plasma concentrations of (Z)-endoxifen greater than $5.97 \mathrm{ng} / \mathrm{mL}$ when compared to those having lower concentrations, thereby permitting a threshold therapeutic concentration to become established for the first time [13]. Similarly, a recent study found that low (Z)-endoxifen levels ( $<14.15 \mathrm{nM} ; 5.29 \mathrm{ng} / \mathrm{mL}$ ) were associated with a shorter period of time free of disease or death as compared to high concentrations of this metabolite (> $35 \mathrm{nM} ; 13.07 \mathrm{ng} / \mathrm{mL}$ ), where the increased risk, expressed as a hazard ratio, was 1.94 [24].

On this basis, it was estimated that over $20 \%$ of women treated with tamoxifen in the standard daily dose of $20 \mathrm{mg}$ may not receive the expected benefits from the applied therapy $[13,26]$. It has also been shown that increasing the daily dose of tamoxifen to 30-40 mg for women with the PM and IM phenotypes leads to increased (Z)-endoxifen plasma concentrations to greater than or close to the threshold value of $5.97 \mathrm{ng} / \mathrm{mL}$ [26-29]. It can thus be assumed that it is possible to increase treatment efficacy in those selected patients with plasma (Z)-endoxifen levels that potentially do not reach therapeutic levels, by suitably tailoring the treatment, (i.e. through type of drug and/or dose), to individual patient requirements.

Studies conducted at the Maria Sklodowska-Curie Memorial Cancer Center and Institute of Oncology in Warsaw on 280 breast cancer patients showed that plasma concentrations of (Z)-endoxifen, as measured by mass spectrometry, in women receiving tamoxifen in the standard $20 \mathrm{mg}$ daily dose is on average $5.55 \mathrm{ng} / \mathrm{mL}$ [15]; this being one of the lowest values of those previously reported from other studies. Moreover, the unexpectedly large group of women surveyed (60\%), achieved (Z)-endoxifen concentrations during their treatment which were lower than the reported therapeutic threshold value of $5.97 \mathrm{ng} / \mathrm{mL}$. This group included over $30 \%$ patients possessing both alleles of the CYP2D6 


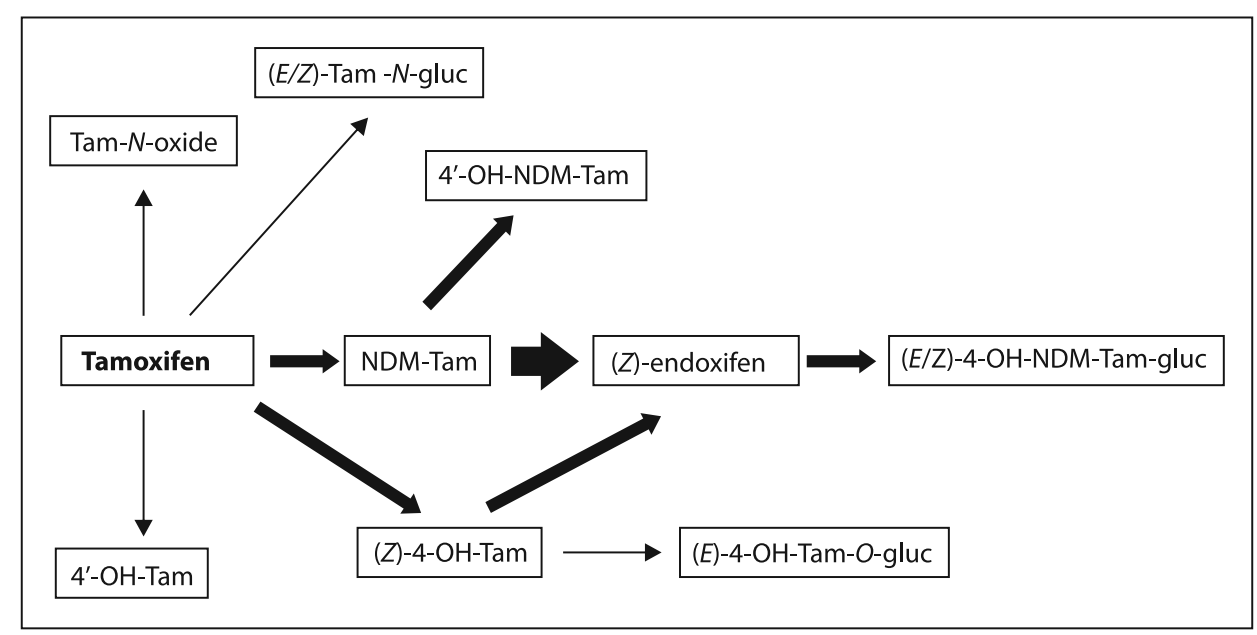

Figure 1. CYP2D6 metabolism of tamoxifen directed towards formation of the active metabolite (Z)-endoxifen. Modified [15]. Tam - tamoxifen; NDM - N-desmethylo-; gluc - glucuronide

gene that encodes the fully functional enzyme. Such findings may indicate that the majority of Polish women treated for breast cancer with the standard dose of the drug, have insufficiently high concentrations of (Z)-endoxifen and therefore will not gain the desired therapeutic effects. Taken together with other studies, these results in particular confirm that determining the CYP2D6 genotype is of marginal significance when making prognoses regarding achievable (Z)-endoxifen concentrations.

\section{Monitoring and personalising treatment}

It is estimated that genetic variability in the CYP2D6gene can only account for $26-40 \%$ of the inter-individual variation in (Z)-endoxifen concentrations achieved in the plasma of women treated with tamoxifen $[6,15,30]$. In contrast, the variability in the extent by which the drug's metabolism is directed towards (Z)-endoxifen formation (Fig. 1), expressed by the ratio of ( $Z$ )-endoxifen to the summed concentrations of tamoxifen and its other metabolites, was observed to be CYP2D6 genotype-dependent in more than $50 \%$ [15]. It was also shown that other genetic factors (including CYP2C9, CYP2C19 and CYP3A5 polymorphisms), as well as non-genetic ones (for instance age and body mass index; BMI) may, by around 3\%, account for the efficiency of converting $\mathrm{N}$-desmethyl-tamoxifen to endoxifen, whilst $53 \%$ is accounted for by the CYP2D6 genotype [24]. Other observations indicate that a more rapid excretion of active metabolites from the body is associated with genetic polymorphism of phase Il enzymes, such as SULTs and UGTs, which may also modify tamoxifen metabolism and affect steady-state concentrations of (Z)-endoxifen [17].

It is currently known that concomitantly taking other medication may also significantly affect steady-state concentrations of active tamoxifen metabolites. Over $30 \%$ of patients treated with tamoxifen are besides treated for de- pression or hot flashes, which are the most common adverse effects of such therapy [17]. Some antidepressants, (like fluoxetine and paroxetine belonging to the selective serotonin reuptake inhibitors), are potent inhibitors of CYP2D6, causing significant decreases in plasma (Z)-endoxifen concentrations $[14,17,20]$. This is especially evident in patients with the EM phenotype, where metabolite concentrations may fall down to levels found even in the PM phenotype. The effects of CYP2D6 inhibitors may in part explain such a low plasma (Z)-endoxifen concentration in patients treated in Poland, where any information on taking other medication is usually fragmentary, typically obtained from the patients themselves and is not systematically monitored.

Another important factor that significantly impacts on the steady-state (Z)-endoxifen concentrations in plasma is patient compliance to the treatment regimen. It has been shown that up to $50 \%$ patients receiving tamoxifen therapy for breast cancer, interrupt their treatment before the five years has expired [31]. Failure to comply with the therapy is more often observed in women with the active CYP2D6 enzyme compared to those with the inactive form. The reason for this is presumed to be the more frequent appearance of distressing side effects of the treatment [32].

The presented facts and observations indicate that personalising treatment could significantly increase the number of patients responding to tamoxifen treatment and thereby reduce its side effects. To this end, it seems that direct monitoring of plasma tamoxifen and its metabolites is the most preferred option for the treated women. Not only does this approach enable determining whether (Z)-endoxifen concentrations reach the therapeutically effective threshold level, but to also assess if low concentrations of this metabolite are due to unfavourable metabolic pathways being preferred (i.e. characterised by a low ratio of (Z)-endoxifen to the summed concentrations of tamoxifen and its other metabolites), or 
indeed for other reasons; such as taking medication(s) that modify enzyme activity or treatment non-compliance by patients (low concentrations of tamoxifen).

Patients with an effective tamoxifen metabolism, burdened with a lower risk of relapse, should be persuaded to strictly comply with the therapy, whilst patients with plasma (Z)-endoxifen concentrations below the therapeutic threshold and an adverse drug metabolism, should be proposed personalised treatment through increasing the daily dose of tamoxifen or, if at all possible, by using an alternative drug. Work is currently undergoing to also determine whether standard doses of tamoxifen can be supplemented with pure (Z)-endoxifen [33]. Any final assertion on whether it is possible to personalise tamoxifen treatment requires separate and adequately controlled multicenter studies. Nevertheless, in the light of the above observations presented, a call for undertaking such research appears fully justified.

\section{Conclusions}

Until now, studies indicate that a significant proportion of breast cancer women undergoing tamoxifen treatment do not achieve sufficiently high plasma concentrations of (Z)-endoxifen, as shown particularly for Polish women, and thus potentially may not share in its therapeutic advantages. The previous proposal of the CYP2D6 genotyping for tamoxifen treated women is of very limited value, in both predicting how effective the treatment will be, as well as knowing whether the therapeutic concentration threshold of (Z)-endoxifen can be attained. In the context of personalising such treatment and its clinical utility, it appears that monitoring steady-state concentrations of tamoxifen and its metabolites is the preferred option.

\section{List of abbreviations used:}

CYP - cytochrome P450

SULT — sulfotransferase

UGT — UDP glucuronosyltransferase

PM - poor metaboliser

IM - intermediate metaboliser

EM - extensive metaboliser

UM - ultra-rapid metaboliser

Conflicts of interest: The author declares no conflict of interest

\section{Prof. Ewa E. Hennig, MD, PhD}

Department of Gastroenterology, Hepatology and Clinical Oncology Medical Center for Postgraduate Education,

Maria Sklodowska-Curie Memorial Cancer Center and Institute of Oncology

Roentgena 5, 02-781 Warsaw, Poland

e-mail:hennige@coi.waw.pl

Received: 1 Mar 2016

Accepted: 14 Apr 2016

\section{References}

1. Veronesi U, Maisonneuve P, Rotmensz N et al. Tamoxifen for the prevention of breast cancer: late results of the Italian Randomized Tamoxifen Prevention Trial among women with hysterectomy. J Natl Cancer Inst 2007; 99: 727-737.

2. Early Breast Cancer Trialists' Collaborative Group (EBCTCG), Davies C, Godwin J, Gray R et al. Relevance of breast cancer hormone receptors and other factors to the efficacy of adjuvant tamoxifen: patient-level meta-analysis of randomised trials. Lancet 2011; 378: 771-784.

3. Davies C, Pan H, Godwin J et al. Long-term effects of continuing adjuvant tamoxifen to 10 years versus stopping at 5 years after diagnosis of oestrogen receptor-positive breast cancer: ATLAS, a randomised trial. Lancet 2013; 381: 805-816.

4. Coezy E, Borgna JL, Rochefort H. Tamoxifen and metabolites in MCF7 cells: correlation between binding to estrogen receptor and inhibition of cell growth. Cancer Res 1982; 42: 317-323.

5. Johnson MD, Zuo H, Lee K-H et al. Pharmacological characterization of 4-hydroxy-N-desmethyl tamoxifen, a novel active metabolite of tamoxifen. Breast Cancer Res Treat 2004; 85: 151-159.

6. Mürdter TE, Schroth W, Bacchus-Gerybadze $L$ et al. Activity levels of tamoxifen metabolites at the estrogen receptor and the impact of genetic polymorphisms of phase I and II enzymes on their concentration levels in plasma. Clin Pharmacol Ther 2011; 89: 708-717.

7. Teunissen SF, Rosing $\mathrm{H}$, Seoane MD et al. Investigational study of tamoxifen phase I metabolites using chromatographic and spectroscopic analytical techniques. J Pharm Biomed Anal 2011; 55: 518-526.

8. Desta Z, Ward BA, Soukhova NV et al. Comprehensive evaluation of tamoxifen sequential biotransformation by the human cytochrome P450 system in vitro: prominent roles for CYP3A and CYP2D6. J Pharmacol Exp Ther 2004; 310: 1062-1075.

9. Robertson DW, Katzenellenbogen JA, Long DJ et al.Tamoxifen antiestrogens. A comparison of the activity, pharmacokinetics, and metabolic activation of the cis and trans isomers of tamoxifen. J Steroid Biochem 1982; 16: 1-13.

10. Rebsamen MC, Desmeules J, Daali Y et al. The AmpliChip CYP450 test: cytochrome P450 2D6 genotype assessment and phenotype prediction. Pharmacogenomics J 2009; 9: 34-41.

11. Sistonen J, Sajantila A, Lao O et al. CYP2D6 worldwide genetic variation shows high frequency of altered activity variants and no continental structure. Pharmacogenet Genomics 2007; 17: 93-101.

12. Stearns $\mathrm{V}$, Johnson MD, Rae JM et al. Active tamoxifen metabolite plasma concentrations after coadministration of tamoxifen and the selective serotonin reuptake inhibitor paroxetine. J Natl Cancer Inst 2003; 95: 1758-1764

13. Madlensky L, Natarajan L, Tchu S et al. Tamoxifen metabolite concentrations, CYP2D6 genotype, and breast cancer outcomes. Clin Pharmacol Ther 2011; 89: 718-725.

14. Borges S, Desta Z, Li L et al. Quantitative effect of CYP2D6 genotype and inhibitors on tamoxifen metabolism: implication for optimization of breast cancer treatment. Clin Pharmacol Ther 2006; 80: 61-74.

15. Hennig EE, Piatkowska M, Karczmarski J et al. Limited predictive value of achieving beneficial plasma (Z)-endoxifen threshold level by CYP2D6 genotyping in tamoxifen-treated Polish women with breast cancer. BMC Cancer 2015; 15: 570.

16. Schroth W, Antoniadou L, Fritz P et al. Breast cancer treatment outcome with adjuvant tamoxifen relative to patient CYP2D6 and CYP2C19 genotypes. J Clin Oncol 2007; 25: 5187-5193.

17. Binkhorst $\mathrm{L}$, Mathijssen $\mathrm{RH}$, Jager A et al. Individualization of tamoxifen therapy: much more than just CYP2D6 genotyping. Cancer Treat Rev 2015; 41: 289-299.

18. de Vries Schultink AH, Zwart W, Linn SC et al. Effects of pharmacogenetics on the pharmacokinetics and pharmacodynamics of tamoxifen. Clin Pharmacokinet 2015; 54: 797-810.

19. Kiyotani $\mathrm{K}$, Mushiroda $\mathrm{T}$, Zembutsu $\mathrm{H}$ et al. Important and critica scientific aspects in pharmacogenomics analysis: lessons from controversial results of tamoxifen and CYP2D6 studies. J Hum Genet 2013; 58: 327-333.

20. Jin Y, Desta Z, Stearns V et al. CYP2D6 genotype, antidepressant use, and tamoxifen metabolism during adjuvant breast cancer treatment. J Nat/ Cancer Inst 2005; 97: 30-39.

21. Wu X, Hawse JR, Subramaniam $M$ et al. The tamoxifen metabolite, endoxifen, is a potent antiestrogen that targets estrogen receptor alpha for degradation in breast cancer cells. Cancer Res 2009; 69: 1722-1727.

22. Hawse JR, Subramaniam M, Cicek M et al. Endoxifen's molecular mechanisms of action are concentration dependent and different than that of other anti-estrogens. PloS One 2013; 8: e54613. 
23. Gong IY, Teft WA, Ly J et al. Determination of clinically therapeutic endoxifen concentrations based on efficacy from human MCF7 breast cancer xenografts. Breast Cancer Res Treat 2013; 139: 61-69.

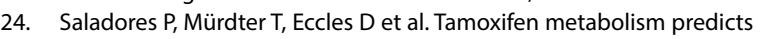
drug concentrations and outcome in premenopausal patients with early breast cancer. Pharmacogenomics J 2015; 15: 84-94.

25. Love RR, Desta Z, Flockhart D et al. CYP2D6 genotypes, endoxifen levels, and disease recurrence in 224 Filipino and Vietnamese women receiving adjuvant tamoxifen for operable breast cancer. Springerplus 2013; 2: 52.

26. Jager NG, Rosing $\mathrm{H}$, Schellens JH et al. Tamoxifen dose and serum concentrations of tamoxifen and six of its metabolites in routine clinical outpatient care. Breast Cancer Res Treat 2014; 143: 477-483.

27. Irvin WJ, Walko CM, Weck KE et al. Genotype-guided tamoxifen dosing increases active metabolite exposure in women with reduced CYP2D6 metabolism: a multicenter study. J Clin Oncol 2011; 29: 3232-3239.

28. Martinez de Dueñas E, Ochoa Aranda E, Blancas Lopez-Barajas I et al. Adjusting the dose of tamoxifen in patients with early breast cancer and CYP2D6 poor metabolizer phenotype. Breast 2014; 23: 400-406.
29. Kiyotani K, Mushiroda T, Imamura CK et al. Dose-adjustment study of tamoxifen based on CYP2D6 genotypes in Japanese breast cancer patients. Breast Cancer Res Treat 2012; 131: 137-145.

30. Antunes MV, Linden R, Santos TV et al. Endoxifen levels and its association with CYP2D6 genotype and phenotype: evaluation of a southern Brazilian population under tamoxifen pharmacotherapy. Ther Drug Monit 2012; 34: 422-431.

31. van Herk-Sukel MP, van de Poll-Franse LV, Voogd AC et al. Half of breast cancer patients discontinue tamoxifen and any endocrine treatment before the end of the recommended treatment period of 5 years: a population-based analysis. Breast Cancer Res Treat 2010; 122: 843-851.

32. Goetz MP, Rae JM, Suman VJ et al. Pharmacogenetics of tamoxifen biotransformation is associated with clinical outcomes of efficacy and hot flashes. J Clin Oncol 2005; 23: 9312-9318.

33. Dickschen $\mathrm{K}$, Eissing $\mathrm{T}$, Mürdter T et al. Concomitant use of tamoxifen and endoxifen in postmenopausal early breast cancer: prediction of plasma levels by physiologically-based pharmacokinetic modeling. Springerplus 2014; 3: 285. 